\title{
Detection of Escherichia coli Strains Producing AmpC Enzymes using Ceftazidime-Imipenem Antagonism Test (CIAT)
}

\author{
Ejikeugwu Chika*1, Duru Carissa $^{2}$, Iroha Ifeanyichukwu' ${ }^{1}$, Oguejiofor Benigna ${ }^{1}$, \\ Okoro Loveday ${ }^{3}$, Ugwu Malachy ${ }^{2}$ \\ ${ }^{1}$ Department of Applied Microbiology, Faculty of Sciences, Ebonyi State University, Abakaliki, \\ Ebonyi State, Nigeria. \\ ${ }^{2}$ Department of Pharmaceutical Microbiology and Biotechnology, Faculty of Pharmaceutical \\ Sciences, Nnamdi Azikiwe University, Awka, Nigeria \\ ${ }^{3}$ Department of Pharmaceutics and Pharmaceutical Microbiology, Ahmadu Bello University, \\ Zaria, Kaduna state, Nigeria
}

\begin{abstract}
Effective monitoring of the development and spread of antimicrobial resistant bacteria including AmpC producing Escherichia coli in abattoir is critical to the containment of disease outbreak due to these microbes. The colonization of slaughtered farm animals with E. coli producing AmpC enzymes and other antibiotic resistant genes portend serious public health implications for the general public - since these organisms are multidrug resistant, and thus remain active even in the face of potent antimicrobial onslaught. This study evaluated the occurrence of AmpC-producing E. coli from abattoir. The isolation of E. coli was done using standard microbiological methods on MacConkey agar and eosin methylene blue (EMB) agar plates; and antimicrobial susceptibility test was done on Mueller Hinton (MH) agar plates using the Kirby-Bauer disk diffusion techniques as per the clinical laboratory standard institute (CLSI) criteria. Screening for the presence of AmpC enzymes in the test isolates was done using a modified double disk approximation method while AmpC enzyme production was phenotypically confirmed using the ceftazidime-imipenem antagonism test (CIAT). A total of $10(32.3 \%)$ E. coli isolates was bacteriologically recovered from thirty one (31) anal swab samples from abattoir. The E. coli isolates showed reduced susceptibility to ertapenem, amikacin, ceftriaxone, imipenem, gentamicin, cefotaxime, meropenem and cefoxitin. In particular, oxacillin had no inhibitory activity against the isolated E. coli isolates. Ciprofloxacin, ofloxacin and ceftazidime showed appreciable inhibitory activity against the E. coli isolates. AmpC enzyme was phenotypically confirmed in $6(60 \%)$ E. coli isolates from abattoir. The findings from this study suggested that animals could function as reservoirs of antibiotic resistant bacteria. It is thus vital to monitor and control the use of antimicrobial agents in the community in order to forestall the emergence and spread of antibiotic resistant bacteria in the environment.
\end{abstract}

Keywords: AmpC enzymes, Escherichia coli, Antimicrobial Resistance, Abattoir, Nigeria

\section{INTRODUCTION}

Food-producing animals occasionally serve as routes or reservoirs for the dissemination of antibiotic resistant bacteria in the community either directly or indirectly because of the presence of antibiotic residues in them. The recurrent and irrational use of antibiotics in animal husbandry and in other agricultural practices allows bacteria to develop and acquire drug resistant genes over time through selective pressure; and this phenomenon accounts for the singular reason there is for the emergence and dissemination of drug resistant bacteria in the community. According to Hyun-Ho and SeungHak ${ }^{[1]}$ antibiotic resistant bacteria pose a significant health problem that could be degenerated through the irrational and continued use of antibiotics in both the community and hospital environment. AmpC enzymes are cephalosporinases encoded on the chromosomes of many Enterobacteriaceae and a few other bacteria where they mediate resistance to cephalothin, cefazolin, cefoxitin and most penicillins. ${ }^{[2,3,4]}$ These enzymes are active on cephamycins; and they are encoded by bacterial chromosomes and plasmids in Gram negative bacilli. According to Jacoby, ${ }^{[5]} \mathrm{AmpC}$ enzyme production in most Enterobacteriaceae including Klebsiella species and Escherichia coli is low but the hyper-production of AmpC enzymes in these organisms is usually induced by the exposure of the bacteria to beta-lactam drugs. The long-term and undue use of antibiotics in livestock, poultry and other agricultural activities selects for drug resistance in pathogenic bacteria including $E$. 
coli. ${ }^{[6,7,8]}$ The increasing global problem of antibiotic resistance in livestock environments has been previously reported. ${ }^{[9,10]}$ Escherichia coli is the most prevalent facultative anaerobic Gram negative bacterial species that inhabit the gastrointestinal tract (GIT) of humans and animals. ${ }^{[1]}$ Though a harmless bacterium, E. coli can become pathogenic through the acquisition of virulent genes including antibiotic resistant genes from its environment; and this makes the organism a medically important bacterium that could cause public health problem if left unchecked. Previous studies have shown that pathogenic bacteria including $E$. coli could be transmitted through the food chain to humans especially through the consumption of contaminated food (meat) products. ${ }^{[11,12,13,14]}$ The wild dissemination of antimicrobial resistance among bacterial populations in the community is an increasing problem worldwide; and this increasing trend needs to be brought under control through periodic monitoring and sustained detection of resistance phenotypes in both the community and hospital environment. Thus, this study investigated the frequency of AmpC-producing Escherichia coli from a local abattoir in Abakaliki metropolis of Ebonyi state, Nigeria.

\section{MATERIALS AND METHOD}

\subsection{Collection of Samples and Processing}

A total of thirty one (31) samples from the anal region of cows from a local abattoir in Abakaliki metropolis, Ebonyi state, Nigeria were used for this study. The samples were collected using sterile swab sticks which were each inserted into the anal region of the cows at a depth of $3 \mathrm{~cm}$ and rotated in a clockwise direction to obtain the sample. The swab sticks were returned to their respective containers, labeled and transported to the microbiology laboratory of Ebonyi State University, Abakaliki for bacteriological analysis. Each of the swab sticks were dipped into test tubes containing $5 \mathrm{ml}$ of freshly prepared nutrient broth (Oxoid, UK) and the tubes were loosely covered with cotton wool. Incubation was carried out at $30^{\circ} \mathrm{C}$ for $18-24$ hours. Bacterial growth was indicated by the presence of turbidity in the tubes.

\subsection{Isolation and Identification of Escherichia coli}

A loopful of the overnight broth culture from the test tubes were aseptically cultured on freshly prepared MacConkey agar (Oxoid, UK) plates and eosin methylene blue (EMB) plates (Oxoid, UK), and these were incubated at $30^{\circ} \mathrm{C}$ for $18-24$ hours. After incubation, suspect colonies of E. coli were sub cultured onto freshly prepared Mac Conkey agar plates and EMB agar plates for the isolation of pure cultures of E. coli. E. coli produces non-mucoid lactose-fermenting colonies on Mac Conkey agar and colonies with metallic sheen on EMB agar. The E. coli isolates were identified using standard microbiological identification techniques. ${ }^{[15]}$

\subsection{Antimicrobial Susceptibility Test}

Antimicrobial susceptibility test was carried out using the Kirby-Bauer disk diffusion method as recommended by the Clinical Laboratory Standard Institute (CLSI) criteria. ${ }^{[16]}$ Commercially available single antibiotic disks (Oxoid, UK) of various antibiotic concentrations including: imipenem (IPM, $10 \mu \mathrm{g}$ ), meropenem (MEM, $10 \mu \mathrm{g}$ ), ertapenem (ETP, $10 \mu \mathrm{g}$ ), amikacin (AK, $10 \mu \mathrm{g}$ ), ofloxacin (OFX, $5 \mu \mathrm{g}$ ) ceftazidime (CAZ, $30 \mu \mathrm{g}$ ), ceftriaxone (CRO, $30 \mu \mathrm{g}$ ), cefotaxime (CTX, $30 \mu \mathrm{g}$ ), ciprofloxacin (CIP, $10 \mu \mathrm{g}$ ), gentamicin (CN, $10 \mu \mathrm{g}$ ), oxacillin (OX, $1 \mu \mathrm{g}$ ), and cefoxitin (FOX, $30 \mu \mathrm{g}$ ) were used. Suspensions of test E. coli isolates (adjusted to $0.5 \mathrm{McFarland}$ turbidity standards) were aseptically swabbed on Mueller-Hinton (Oxoid, UK) agar plates using swab sticks; and each of the antibiotic disks was placed on the plate at a distance of $15 \mathrm{~mm}$. Susceptibility test plates were incubated at $30^{\circ} \mathrm{C}$ for $18-24 \mathrm{hrs}$; and the inhibition zone diameters were recorded and interpreted as per the recommendations of CLSI. ${ }^{[7,16]}$ Quality control for susceptibility study was achieved by using standard strain of E. coli ATCC 25922.

\subsection{Amp C Screening Test}

A modified double disk approximation method (MDDM) was used to screen the E. coli isolates for the presence of AmpC enzymes according to the method of Singhal et al. ${ }^{[17]}$ and Cantarelli et al. ${ }^{[18]} \mathrm{A}$ suspension of the test $E$. coli isolate (adjusted to $0.5 \mathrm{McFarland}$ turbidity standards) was swabbed on Mueller-Hinton agar plates and antibiotic disk of cefotaxime $(30 \mu \mathrm{g})$ and ceftazidime $(30 \mu \mathrm{g})$ were placed adjacent to cefoxitin $(30 \mu \mathrm{g})$ disk at a distance of $20 \mathrm{~mm}$ from each other. Bacterial isolates showing blunting of ceftazidime or cefotaxime zone of inhibition adjacent to cefoxitin disk or showing reduced susceptibility to either of ceftazidime or cefotaxime and cefoxitin were considered possible AmpC enzyme producers; and these were selected for detection of AmpC enzymes using the ceftazidime-imipenem antagonism test (CIAT). 
Detection of Escherichia coli Strains Producing AmpC Enzymes using Ceftazidime-Imipenem Antagonism Test (CIAT)

\subsection{Ceftazidime-Imipenem Antagonism Test (CIAT)}

CIAT was performed using ceftazidime $(30 \mu \mathrm{g})$ disk, cefoxitin $(30 \mu \mathrm{g})$ disk and imipenem disk (10 $\mu \mathrm{g})$ as described by Cantarelli et al. ${ }^{[18]}$ Ceftazidime disc and imipenem disk were placed at a distance of $20 \mathrm{~mm}$ apart on Mueller-Hinton agar plate previously inoculated with a suspension of the test bacteria (adjusted to 0.5 McFarland turbidity standards) that showed reduced susceptibility to cefoxitin. A cefoxitin disk $(30 \mu \mathrm{g})$ was also placed at a distance of $20 \mathrm{~mm}$ from the ceftazidime disk for comparison. All the susceptibility test plates were incubated at $30^{\circ} \mathrm{C}$ for $18-24 \mathrm{hrs}$. Antagonism indicated by a visible reduction in the inhibition zone around the ceftazidime disk adjacent to the imipenem or cefoxitin disk was inferred as a positive inducible AmpC beta-lactamase production. ${ }^{[18]}$

\section{RESULTS}

The frequency of isolation of Escherichia coli in this study is shown in Table 1. Out of the thirty one (31) anal swab samples of cow bacteriologically analyzed in this study, Escherichia coli was only isolated from 10 anal swab samples (Table 1). Biochemically, the isolated E. coli isolates which were Gram negative rods were found to be positive to indole test and methyl red (MR) test. Table 2 shows the antimicrobial susceptibility profile of the isolated $10 \mathrm{E}$. coli isolates to some selected antibiotics. The isolated E. coli showed varying levels of susceptibility and resistance to the tested antibiotics as indicated by their respective inhibition zone diameter (IZD).

Table1. Frequency of Escherichia coli Isolation

\begin{tabular}{|c|c|c|c|}
\hline Sample $(\mathbf{n}=31)$ & Source & Organism & No (\%) of $\boldsymbol{E}$. coli isolated \\
\hline Anal swabs of cow & Abattoir & Escherichia coli & $10(32.3)$ \\
\hline
\end{tabular}

Table2. Inhibition Zone Diameter (IZD) of the Isolated Escherichia coli

\begin{tabular}{|c|c|c|c|c|c|c|c|c|c|c|c|c|}
\hline Isolates & AK & CRO & FOX & CAZ & CN & OFX & MEM & IPM & ETP & CTX & CIP & OX \\
\hline E1 & 10 & 10 & 14 & 17 & 11 & 20 & 25 & 14 & - & - & 25 & - \\
\hline E2 & 17 & 11 & 11 & 24 & 16 & 22 & 20 & 18 & 17 & - & 21 & - \\
\hline E3 & 17 & 18 & - & 11 & 25 & 21 & 18 & 18 & 12 & - & 25 & - \\
\hline E4 & 13 & 12 & - & 10 & 13 & 18 & 14 & 16 & - & - & 18 & - \\
\hline E5 & 16 & 4 & - & - & - & - & 29 & 18 & 18 & - & 18 & - \\
\hline E6 & - & 9 & 15 & 16 & 10 & 10 & 14 & 16 & - & - & - & - \\
\hline E7 & 8 & 12 & 12 & 20 & 10 & 12 & - & - & - & 12 & - & - \\
\hline E8 & 10 & 20 & 15 & 12 & 9 & 11 & - & 10 & - & 10 & - & - \\
\hline E9 & 12 & 18 & 16 & 11 & 6 & 20 & - & - & 12 & 20 & 10 & - \\
\hline E10 & 9 & 6 & 10 & 10 & 8 & 11 & - & - & 10 & - & 12 & - \\
\hline
\end{tabular}

Key: IPM=imipenem, $M E M=$ meropenem, ETP=ertapenem, $A K=$ amikacin, $O F X=$ ofloxacin, $C A Z=$ ceftazidime, $C R O=$ ceftriaxone, $C T X=$ cefotaxime, $C I P=$ ciprofloxacin, $C N=$ gentamicin, $O X=$ oxacillin, $F O X=$ cefoxitin

In particular, higher levels of resistance was recorded amongst the isolated $E$. coli isolates to oxacillin, cefotaxime, meropenem and cefoxitin - to which most of the test $E$. coli isolates showed least susceptibility to. Oxacillin had no inhibitory activity against the isolated $E$. coli isolates used in this study (Table 2). The prevalence of AmpC-producing E. coli isolates from the isolated E. coli isolates screened for- and confirmed phenotypically for the production of AmpC enzymes is shown in Table. 3. Out of the $10 \mathrm{E}$. coli isolates screened and tested phenotypically for the production of AmpC enzymes by the ceftazidime-imipenem antagonism test (CIAT), only six (6) E. coli isolates were phenotypically confirmed to produce AmpC enzymes. AmpC enzymes was not confirmed in the other four (4) E. coli isolates since they showed susceptibility to cefoxitin - for which AmpC-producing bacteria are usually resistant to (Table 3 ).

Table3. Occurrence of AmpC-producing Escherichia coli

\begin{tabular}{|c|c|c|c|r|r|}
\hline Sample & Source & No (\%) of samples & No (\%) of E. coli screened & $\begin{array}{c}\text { AmpC } \\
\text { Positive }\end{array}$ & $\begin{array}{c}\text { AmpC } \\
\text { negative }\end{array}$ \\
\hline Anal swabs cow & Abattoir & 31 & $10(32.3)$ & $6(60)$ & $4(40)$ \\
\hline
\end{tabular}

\section{DisCUSSION}

The unnecessary and inappropriate use of antibiotics in rearing of animals, poultry birds and in other veterinary or agricultural purposes favours the emergence and spread of resistant bacteria in the community through selective pressure. And the wild reservoirs of antibiotic resistant bacteria in 


\section{Ejikeugwu Chika et al.}

animals is critical to human health - owing that antimicrobials are used for the management and treatment of microbial related diseases in man. And the selective pressure caused by the irrational use of antimicrobial agents in husbandry, veterinary and agricultural practices as reported by Radhouani et $a l .{ }^{[14]}$ is considered as one of the major factor responsible for the emergence and spread of drugresistant bacteria in the community. This study evaluated the occurrence of AmpC-producing Escherichia coli from abattoir. The result of this study show that only $10 \mathrm{E}$. coli isolates was recovered from the 31 anal swab samples from anal region of cows in a local abattoir in Abakaliki metropolis, Ebonyi State, Nigeria. E. coli is a member of the Enterobacteriaceae family that is commonly found in the gastrointestinal tract of animals and humans. ${ }^{[19]}$ But pathogenic strains of $E$. coli especially those harbouring antibiotic resistance genes could pose serious health challenge since they may resist the effect of antimicrobial agents directed against them. According to Cho et al., ${ }^{[8]}$ Escherichia coli strains resistant to antibiotics are harboured by farm animals, farm workers and poultry birds. The same study by Cho et al also reported higher resistant rates of the isolated $E$. coli to some commonly used antibiotics. ${ }^{[8]}$ According to Van Den Bogaard et al. ${ }^{[20]}$, resistant feacal E. coli from farm animals including poultry birds can infect humans both directly and indirectly through food by the colonization of the human intestinal tract by these organisms - which contribute drug resistant genes to the human endogenous normal microflora. ${ }^{[20]}$ Also in this study, we examined the antimicrobial resistance profile in the $E$. coli isolated from the anal swab samples from abattoir. The isolated E. coli were found to be mostly resistant to cefoxitin, cefotaxime, ertapenem, amikacin, and ciprofloxacin. However, the inhibition zone diameter produced by the test $E$. coli isolates to the tested antibiotics showed that the organism was susceptible to ciprofloxacin and amikacin - which are fluoroquinolones and aminoglycosides respectively. Ceftriaxone, ceftazidime and meropenem also showed some level of antimicrobial activity against the $E$. coli isolates. The result of antimicrobial susceptibility of $E$. coli from anal swabs of cow in this study corresponds to the report by Cho et al. ${ }^{[8]}$ - who also reported that $E$. coli from swine and farm animals exhibit varying levels of resistance to both beta-lactam and non-beta-lactam antibiotics. Fischer et al. ${ }^{[12]}$ also showed in their study that $E$. coli from farm animals are multiply resistant to antibiotics inclusive of those used in human medicine. Hyun-Ho and Seung-Hak ${ }^{[1]}$ also opined that E. coli from community samples show reduced susceptibility to some commonly available antimicrobial agents - as obtainable in this present study. We screened for the possible production of AmpC enzymes by the E. coli isolates and also confirmed AmpC enzyme production phenotypically in the test isolates using the ceftazidime-imipenem antagonism test (CIAT). It was discovered in this study that only $6(60 \%) E$. coli isolates were AmpC positive out of the 10 isolates of E. coli phenotypically tested for AmpC enzyme production. The prevalence of AmpC positive E. coli in this study corresponds to the study by Hemalatha et al. ${ }^{[4]}$ who reported AmpC positive Enterobacteriaceae by an inhibition-based method in their study. Higher prevalence's of AmpC producers as obtainable in this study was also reported by Akujobi et al. ${ }^{[21]}$ in Enterobacteriaceae particularly in E. coli and Klebsiella species from human samples. Conclusively, pathogenic bacteria including antibiotic resistant pathogenic E. coli in food producing animals are usually spread through the food chain because of the presence of antibiotic residues in food. This portends grave danger to human health, and thus jeopardizes the efficacy of some available antibiotics used to treat some bacterial-related infections. The occurrence of AmpC-producing E. coli from abattoir calls for effective monitoring and possibly a ban on the use of antibiotics in the production and/or rearing of food producing animals in order to assuage the emergence and spread of drug resistant bacteria in the community. The findings from this presumptive study also support the need for a total review of the use of antibiotics in livestock production and other veterinary purposes in Nigeria so that proper steps will be taken to contain the growing dilemma of antibiotic resistance in this part of the world.

\section{REFERENCES}

[1] Hyun-Ho S and Seung-Hak C (2013). Prevalence of antimicrobial resistance in Escherichia coli strains isolated from fishery workers. Osong Public Health Res Perspect., 4(2):72-75.

[2] Jacoby G.A and Munoz-Price L.S (2005). Mechanism of disease. The New $\beta$ - lactamases. New England Journal of Medicine, 352:380-391.

[3] Perez-Perez F.J and Hanson N.D (2002). Detection of Plasmid - Mediated Amp C beta lactamase genes in clinical isolates by using multiplex PCR. Journal of Clinical Microbiology, 40:2153-2162. 
[4] Hemalatha V., Padma M., Sekar U., Vinodh T.M and Arinkumar A.S (2007). Detection of AmpC beta lactamases production in Escherichia coli and Klebsiella by an inhibitor based method. Indian J. Med Res, 126:220-223.

[5] Jacoby G.A (2009). AmpC $\beta$-lactamases. Clinical Microbiology Reviews, 22(1):161-182.

[6] Ejikeugwu C, Edeh C, Iroha I, Orji J, Eluu S, Ugbo E, Duru C, Esimone C (2016a). Antibiogram and Detection of Metallo-Beta-Lactamase (MBL) positive Escherichia coli isolates from abattoir. Nature and Science, 14(11):65-69.

[7] Ejikeugwu C, Duru C, Iroha I, Ugwu M, Nwakaeze E, Oguejiofor B, Esimone C, Adikwu M (2016b). Phenotypic Screening of Clinical Isolates of Escherichia coli, Klebsiella pneumoniae and Pseudomonas aeruginosa for Metallo-Beta-Lactamase. Life Science Journal, 13(10):77-82.

[8] Cho S-H, Lim Y-S and Kang Y-H (2012). Comparison of antimicrobial resistance in Escherichia coli strains isolated from healthy poultry and swine farm workers using antibiotics in Korea. Osong Public Health Res Perspect, 3(3): 151-155.

[9] Teuber M (2001). Veterinary use and antibiotic resistance. Curr Opin Microbiol., 4(5):493-499.

[10] Miles T.D, McLaughlin W and Brown P.D (2006). Antimicrobial resistance of Escherichia coli isolates from broiler chickens and humans. BMC Veterinary Research, 2(7):1-9.

[11] Rasheed M.U, Thajuddin N, Ahamed P, Teklemariam Z and Jamil K (2014). Antimicrobial drug resistance in strains of Escherichia coli isolated from food sources. Rev. Inst. Med. Trop. Sao Paulo, 56(4):341-346.

[12] Fischer J, Rodriguez I, Schmoger S, Friese A, Roesler U, Helmuth R and Guerra B (2012). Escherichia coli producing VIM-1 carbapenemase isolated on a pig farm. The Journal of Antimicrobial Chemotherapy, 67, 1793-1795.

[13] Gijon D, Curiao T, Baquero F, Coque T.M and Canton R (2012). Fecal carriage of carbapenemase-producing Enterobacteriaceae: a hidden reservoir in hospitalized and nonhospitalized patients. Journal of Clinical Microbiology, 50, 1558-1563.

[14] Radhouani H, Silva N, Poeta P, Torres C, Correia S and Igrejas G (2014). Potential impact of antimicrobial resistance in wildlife, environment, and human health. Frontiers in Microbiology, $5: 1-12$.

[15] Cheesbrough M (2006). District Laboratory Practice in Tropical Countries. $2^{\text {nd }}$ edition. Cambridge University Press, UK. Pp. 178-187.

[16] Clinical Laboratory Standard Institute, CLSI (2011). Performance standards for antimicrobial disk susceptibility test. Fifteenth informational supplement, CLSI document M100-S15. Wayne, PA. USA.

[17] Singhal S, Mathur T, Khan S, Upadhyay D.J, Chugh S, Gaind R and Rattan A (2005). Evaluation of Methods for AmpC Beta-Lactamase in Gram Negative Clinical Isolates from Tertiary Care Hospitals. Indian J Med Microbiol, 23:120-124.

[18] Cantarelli V.V, Inamine E, Brodt T.C.Z, Secchi C, Cavalcante B.C and Pereira F de S (2007). Utility of the ceftazidime-imipenem antagonism test (CIAT) to detect and confirm the presence of inducible AmpC beta-lactamases among Enterobacteriaceae. BJID, 11(2):237-239.

[19] Brooks G.F., Butel J.S and Morse S.A (2004). Medical Microbiology, $23^{\text {rd }}$ edition. McGraw Hill Publishers. USA. Pp. 248-260.

[20] Van Den Bogaard A.E, London N, Driessen C, Stobberingh E.E (2001). Antibiotic resistance of faecal Escherichia coli in poultry, poultry farmers and poultry slaughters. J. Antimicrob. Chemother, 47:763-771.

[21] Akujobi C.O, Odu N.N and Okorondu S.I (2012). Detection of AmpC beta lactamases in clinical isolates of Escherichia coli and Klebsiella. African Journal of Clinical and Experimental Microbiology, 13(1):51-55. 